\title{
Calcium and its Localization in Human Lens Fibres: An Electron Tomographic Study
}

\author{
J. VANMARLE ${ }^{a *}, R$. JONGES ${ }^{b}, G$. F. J. M. VRENSEN" AND A. DEWOLFc \\ Academic Medical Center, University of Amsterdam, ${ }^{\mathrm{a}}$ Departments of Electron Microscopy and ${ }^{\mathrm{b}}$ Medical \\ Physics and Informatics P.O. Box 22700, 1100 DE, Amsterdam \\ and ${ }^{\mathrm{C}}$ Department of Morphology, The Netherlands Ophthalmic Research Institute, P.O. Box 12141, \\ 1100 AC, Amsterdam, The Netherlands
}

(Received Oxford 28 June 1996 and accepted in revised form 27 February 1997)

\begin{abstract}
The ultrastructural distribution of calcium was studied in human lens fibres with the oxalate pyroantimonate technique. In the intermediate cortex precipitates were found perimembranous and occasionally in the fibre cytoplasm. The improved resolution of electron tomography revealed (a) that the perimembranous precipitates as described earlier by Vrensen et al. (1995) are restricted to the intercellular space; no indications were found of an increased intracellular submembranous calcium level, and (b) the existence of an intracellular pool of small protein attached precipitates in the fibre cytoplasm not observed with conventional electron microscopy. It is concluded that in the intermediate and deep cortex, where in the mature fibres all cellular calcium pools have disappeared, two calcium pools exist: (a) a pool of free calcium ionically bound to the negatively charged phospholipids of the external face of the fibre membrane and (b) a cytoplasmic pool of protein associated calcium. Possible candidates for this cytoplasmic calcium binding are discussed. $\quad$ (c) 1977 Academic Press Limited

Key words: human lens; $\mathrm{Ca}^{2+}$ localization; electron tomography; ultrastructure; membranes; $\beta$ crystallins.
\end{abstract}

\section{Introduction}

Calcium is present in the lens in a high concentration $(0 \cdot 5-1 \mathrm{~mm})$, but only a small fraction of it in the free or ionic form $(0 \cdot 1-2 \mu \mathrm{M})$. As in all other cells in lens epithelium and in the superficial fibres, calcium is involved in cell-signalling, but the presence of large amounts of free calcium ions leads to opacities in the lens (Duncan, Williams and Riach, 1994). Therefore an efficacious system to restrict their movement is essential to maintain the transparency of the lens. Measurements with calcium sensitive electrodes revealed a pCa of approximately $6 \cdot 5-6 \cdot 8$ in the superficial cortex. However, approximately $0.5 \mathrm{~mm}$ from the periphery of the lens the pCa dropped abruptly from $6 \cdot 5-6 \cdot 8$ to $4 \cdot 5-4 \cdot 7$, to increase again at $0.8 \mathrm{~mm}$ to 6.5 ; it remains at this level in the deep cortex and nucleus. The method used does not discriminate between the concentrations of inter- and intracellular free calcium (Duncan and Jacob, 1984), but most likely the pCa reflects the intracellular free calcium. No reliable data on the intercellular concentration of calcium are available. Vrensen et al. $(1995,1996)$ used the oxalate-pyroantimonate (OPA) technique for EM localization of free or ionically bound calcium (VanReempts, Borgers and Offner, 1982) in normal rat and human lenses. It was observed in rat and human lenses that in the epithelium and superficial cortex calcium precipitates are scanty and

\footnotetext{
* Correspondence to: J. van Marle, Academic Medical Center, Department of Electron Microscopy, Meibergdreef 15, $1105 \mathrm{AZ}$ Amsterdam, The Netherlands.
}

if present at all they are restricted to mitochondria, to the cisterns of the endoplasmic reticulum and Golgi zone and to the nuclear envelope. In an intermediate or transitional cortical zone calcium precipitates are frequent. They are found in the nuclear envelope, in the cisternae of the endoplasmic reticulum and along the fibre limiting membranes. Gap junctions between these intermediate fibres are consistently devoid of precipitates. These dense accumulations of calcium precipitates co-localize with the region in the lens where (a) the $\mathrm{pCa}$ as measured by Duncan and Jacob (1984) is decreased, where (b) cell organelles and nuclei disappear in the final stages of lens cell differentiation (Basnett and Beebe, 1992) and where (c) the lens fibres become uncoupled (Vrensen et al., 1992; Prescott et al., 1994). The cytoplasmic calcium precipitates rapidly disappear towards the deep cortex. The perimembranous precipitates disappear more gradually, but eventually in the deep cortex and nucleus they are completely absent. Old human lenses with incipient opacities frequently show 'hot Ca-spots' i.e. local accumulations of calcium precipitates in the cytoplasm of affected superficial and deep cortical fibres (DeWolf and Vrensen, 1995).

The calcium pyroantimonate precipitates are small (approximately $5-15 \mathrm{~nm}$ in diameter) even along the lens fibre membranes where the most conspicuous pyroantimonate aggregates are found, and therefore the exact localization of these precipitates as well as their position related to the ultrastructure of the lens fibres cannot be determined with sufficient certainty in a conventional EM approach, since the precision of 

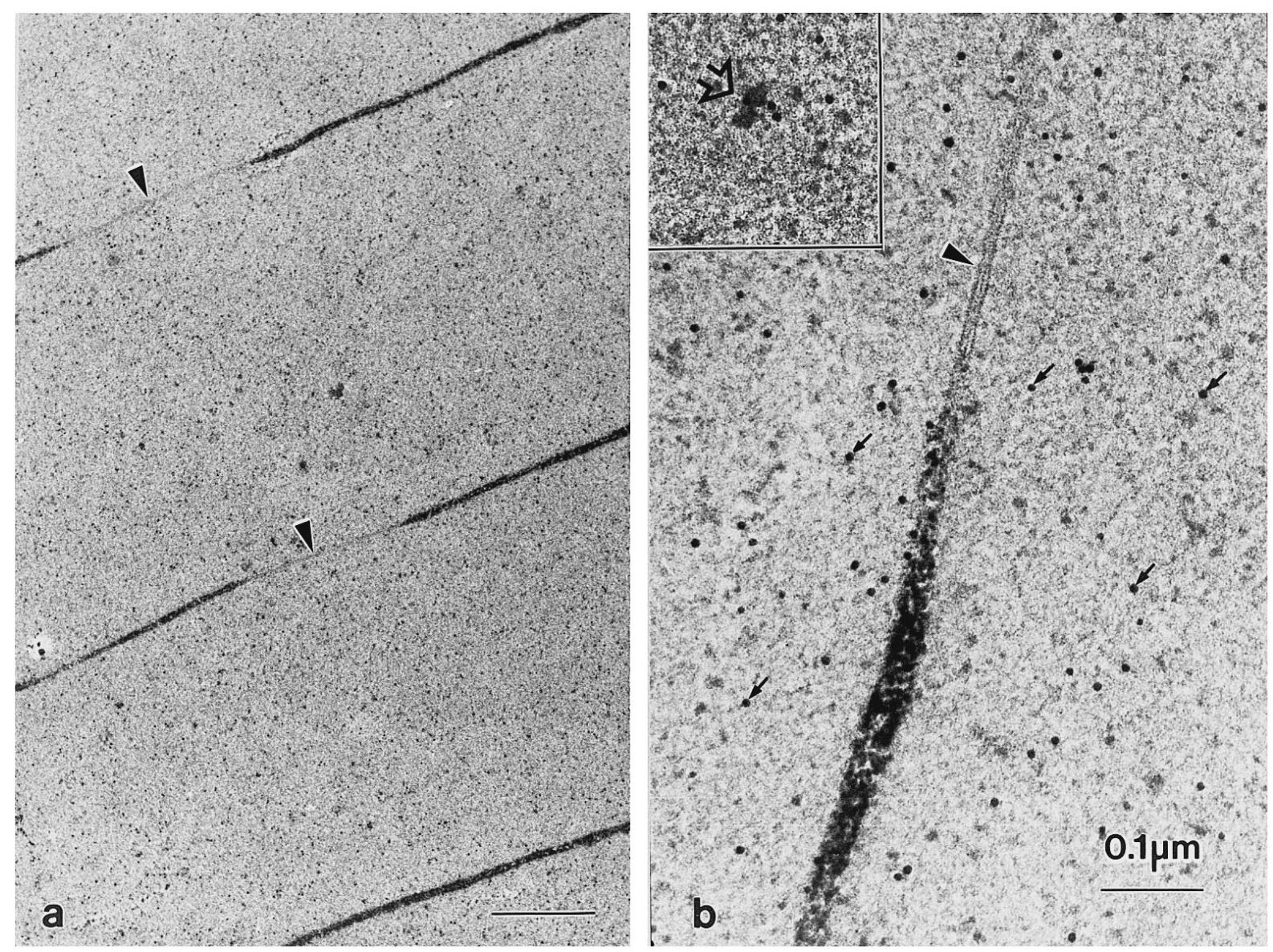

FIG. 1. Presence of calcium pyroantimonate precipitates found between the cortical fibres of a human lens observed with conventional transmission electron microscopy. (a) Overview (scale: 0.5 $\mu \mathrm{m}$ ). The gap junctions (arrowheads) stand out owing to the absence of precipitate $(\mathrm{a}, \mathrm{b})$. Occasionally, precipitates are found in the fibre cytoplasm [(b) inset, open arrow]. The small black dots [some are indicated by small arrows in (b)] are the gold particles used for the alignment of the tilt series. Inset and (b) have identical magnifications.

localization depends on the relation between section thickness and the dimensions of the structures involved. In order to obtain a better understanding of the localization of the calcium precipitates we used a combination of tilt series and electron tomography (a technique fully covered in Frank, 1992) to reconstruct the mass (i.e. scattering material) distribution inside the volume of the $100 \mathrm{~nm}$ epoxy resin section used for conventional electron microscopy. It enabled us to study the distribution of the precipitates as well as their localization in relation to the morphology of the fibre in much more detail. This recently developed technique demonstrated that the perimembranous calcium precipitates are restricted to the intercellular space and visualized a separate calcium pool in the fibre cytoplasm. This cytoplasmic pool was missed in conventional EM studies owing to the small size of the cytoplasmic precipitates $(\leqslant 2 \mathrm{~nm})$.

\section{Material and Methods}

In previous studies on clear human lenses the calcium

distribution was consistent and did not show qualitative variations. Considering the large amount of work necessary for one reconstruction we restricted our reconstructions to three clear human donor lenses (ages 37, 63 and 79 years) which were obtained from the Cornea Bank Amsterdam and treated for calcium detection as described by VanReempts et al. (1982) and Vrensen et al. (1995). Briefly, the lenses were fixed in an oxalate containing glutaraldehyde fixative, and subsequently postfixed in a pyroantimonate containing $\mathrm{OsO}_{4}$ solution. This incubation converts the non-scattering Ca-oxalate into electron scattering Ca-pyroantimonate. After dehydration through graded ethanols and 1,2-propylene oxide and subsequent embedding in epoxy resin $100 \mathrm{~nm}$ sections were cut, collected on 75 mesh grids and contrasted with uranyl acetate and lead citrate. To facilitate computer alignment of the tilt series used for reconstruction, and to determine the thickness of the section, colloidal gold particles $(7 \mathrm{~nm}$ and $10 \mathrm{~nm}$, respectively) were applied before and after collecting the section. To improve attachment of colloidal gold, the specimens were coated with carbon. Tilt series were made with an instrument magnification of 15000 from $+60^{\circ}$ to $-60^{\circ}$ with an increment of $5^{\circ}$, and after rotation of the specimen over $90^{\circ}$ an additional tilt series was made from $+50^{\circ}$ to $-50^{\circ}$ 
with an increment of $10^{\circ}$. A Philips EM420 electron microscope equipped with rotation holder and goniometer stage was used. The micrographs were digitized with a Photometrics cooled CCD camera (12 bit) with $1 \mathrm{~nm}$ set to 1 pixel. Alignment of the tilt series (either over 1 or over 2 tilt axes) and reconstruction (based on back projection and ART) were performed with in-house developed software (Jonges et al., 1996) running on an IBM R6000 type 7012/32H workstation and an IBM 9071SP1 super computer. The reconstructions made from data sets over two tilt axes showed a significant improvement in detail over reconstructions made from data sets limited to one tilt axis. The results, i.e. the reconstructed epoxy resin sections, are presented as a volume allowing the presentation of any section plane through this reconstructed volume (X and Y present the object plane, $\mathrm{Z}$ presents the electron optical axis). Owing to computer presentation of the results, mass in the reconstructed cross sections is invariably indicated as white, the absence of mass as black.

Although the reconstruction yields a highly reliable representation of the mass distribution within the epon section, it is preferable to use the middle layer of the reconstructed volume (approx. 60\%), since the upper and lower part of the epon section show a nonlinear collapse due to working conditions (i.e. vacuum and/or electron exposure) in the electron microscope (VanMarle et al., 1995).

\section{Results}

Conventional TEM imaging has demonstrated perimembranous calcium pyroantimonate precipitates and, occasionally, precipitates in the cytoplasm of the intermediate cortical fibres as described in previous papers [Figs 1(a), and (b) inset] (cf. Vrensen et al., 1995 ; 1996). The gap junctions have been consistently found to be free of precipitate [Figs 1(a), (b)].

It was not possible, using conventional TEM imaging, to observe whether the membrane associated calcium precipitates were restricted to the intercellular space, or whether membrane associated calcium in the cytoplasm was also involved. This problem always occurs with small objects (in this case $10 \mathrm{~nm}$ ) embedded in sections which are thick $(100 \mathrm{~nm})$ in relation to the object size. However, electron tomographic reconstruction of these precipitates and surrounding cytoplasm consistently demonstrated scattering material protruding into the intercellular space with flat surfaces delineating the fibre cytoplasm (Fig. 2). This observation holds for reconstructed planes parallel with the epoxy resin section (XY planes) as well as for $\mathrm{XZ}$ or $\mathrm{YZ}$ section planes [Figs 2(b), (c)]. Therefore, it seems unlikely that the membrane bound cytoplasmic calcium stores contribute to these precipitates. The amount of electron scattering precipitate in the intercellular space varies along the fibre; usually between $30 \%$ to $80 \%$ of the
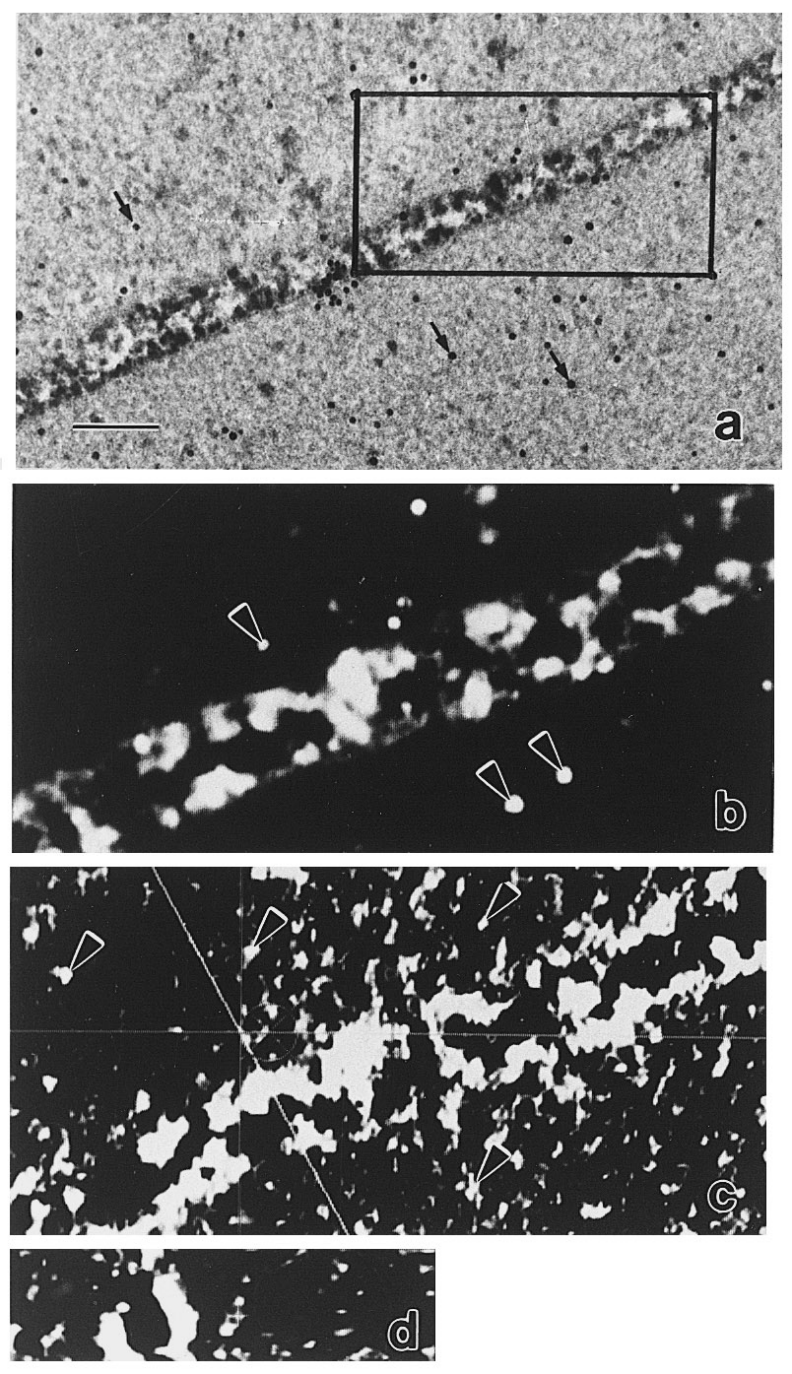

Fig. 2. Conventional electron-microscopic imaging (a) and electron tomographic reconstructions (b, c, d) of intercellular Ca precipitates between the cortical fibres. (a) Conventional imaging of the cortical intercellular precipitates. The frame indicates which part of the section is reconstructed (some gold particles are indicated by small arrows); scale: $100 \mathrm{~nm}$; (b) Simulated electron microscopic image made of all the reconstructed XY planes demonstrating the improved image compared with conventional imaging (the white circular structures (arrowheads) are gold markers used for the alignment of the tilt series); (c) XY plane through the reconstructed volume demonstrating the sharp demarcation of the intercellular precipitates along the fibre membrane; (d) YZ plane through the reconstructed volume along the white line in (c) demonstrating that in this direction the intercellular precipitates are also restricted to the intercellular space and do not extend into the cytoplasm.

Arrow heads in (c) indicate some of the small highly scattering Ca precipitates associated with proteins which are missed using conventional TEM imaging.

Note: Owing to computer presentation of the results, mass in the reconstructed cross sections is invariably represented as white, the absence of mass as black. $\mathrm{X}$ and $\mathrm{Y}$ indicate the orientation of the object plane in the electron microscope, $\mathrm{Z}$ the direction of the electron optical axis (i.e. perpendicular to the XY plane). 
intercellular space is filled with precipitate. Another finding missed, using conventional EM imaging, was the observation that the centre of the intercellular space irrespective of the amount of precipitate was largely free of scattering material; precipitates crossing the intercellular space were found only occasionally. This phenomenon is preserved optimally in the middle layer of the reconstructed volume since this part is not affected by the section collapse due to working conditions in the electron microscope (vacuum and/or electron exposure).

Unexpected, since they were not observed using conventional electron microscopy, were the numerous, seemingly randomly distributed, small (approximately $2 \mathrm{~nm}$ ) highly scattering elements in the fibre cytoplasm (Figs 2 and 3) which were identified as very small calcium pyroantimonate precipitates. In a number of cases they were found in close association with less electron dense irregularly shaped structures representing proteins. The small precipitates were never observed associated with the fibre membranes, nor was their incidence increased in the cytoplasm bordering the membrane. In addition, large calcium precipitates were found incidentally in the cytoplasm. Reconstruction of these large precipitates revealed that they were irregularly shaped and were found throughout the total thickness of the resin section (Fig. $3)$. The amount of small electron scattering precipitates in the direct vicinity of these large precipitates seemed diminished in comparison with those found elsewhere in the cytoplasm. An association with proteins as observed with the small precipitates was absent.

\section{Discussion}

Using electron tomographic reconstructions, we consistently found that the perimembranous precipitates along the fibres as described by Vrensen et al. $(1995,1996)$ were restricted to the intercellular space between the fibres since these precipitates were consistently observed to be smooth at the fibre cell membrane side irrespective of the orientation of the cross section through the reconstructed volume. The precipitates always protruded into the intercellular space, and in most cases the centre of the intercellular space was free of precipitate. These observations warrant the conclusion that the intercellular pool of calcium is the only calcium source involved in the formation of the perimembranous precipitates, whereas submembranous intracellular calcium is not involved.

The presence of a calcium gradient over the intercellular space with the highest concentration close to the fibre membrane and the relative absence of precipitate in the middle of the intercellular space was an unexpected observation. The highest calcium concentration being close to the membrane is in accordance with the large amount of negatively charged phosphate groups of the phospholipids in the fibre membrane which will maintain this gradient. These phosphate groups will bind ionically the $\mathrm{Ca}^{2+}$ ions and inhibit their diffusion into the intercellular space. The presence of a gradient between the membranes in the intercellular space cannot readily be explained as a technical artefact. Rinsing away of small precipitates seems unlikely since the trapping reactions are performed on whole lenses and the intercellular precipitates are formed $0.5 \mathrm{~mm}$ from the outside of the lens and the intercellular precipitates always show this gradient. The relative emptiness of the centre of the intercellular space as a consequence of recrystallization of small precipitates formed in the middle of the intercellular space is also possible, however, the distribution of small precipitates distributed along the fibre membrane seems to invalidate this argument.

The sharp demarcation of the intercellular precipitates along the fibre membrane also indicates that these membranes even in the deeper cortex, in spite of the reduced amount of intermembranous particles (Vrensen et al., 1992) and the increased amount of cholesterol (Duindam et al., 1995, 1996; Li, So and Spector, 1985; VanMarle, Vrensen and VanVeen, 1991) are still able to maintain a gradient.

The small $(2 \mathrm{~nm})$ pyroantimonate particles in the fibre cytoplasm are missed with conventional electron microscope imaging owing to their small size and to the small amount of scattering material in the particles in relation to the total amount of scattering material (epoxy resin as well as osmicated and uranyl/lead contrasted proteins) over the whole thickness of a $100 \mathrm{~nm}$ section. However, in the reconstructed cross sections the particles can be distinguished from the less scattering proteins and from the background of the embedding material. An association with membranes of a higher concentration close to the fibre membranes was not observed, but in many cases these small precipitates are associated with structures characterized by less scattering and irregular shapes: i.e. proteins. This would mean that the submembranous calcium stores in the cortical fibres have disappeared, but it is more likely that the binding of calcium to the calcium binding proteins is so tight that these calcium stores are not detected with the OPA technique (VanReempts et al., 1982).

Considering the proteins with which the small precipitates are associated, there seem to be two possibilities: (a) the calcium binding proteins e.g. calmodulin, calpastatin, calbindin etc. and (b) the $\beta$ crystallins, recently shown to have calcium binding properties (Sharma and Balasubramanian, 1996). The calcium binding proteins do not seem likely candidates as main site for calcium binding since (a) they all bind calcium so tightly that it cannot be demonstrated with the OPA technique (VanReempts et al., 1982), (b) they are often membrane associated and we do not find the small precipitates preferentially associated with mem- 

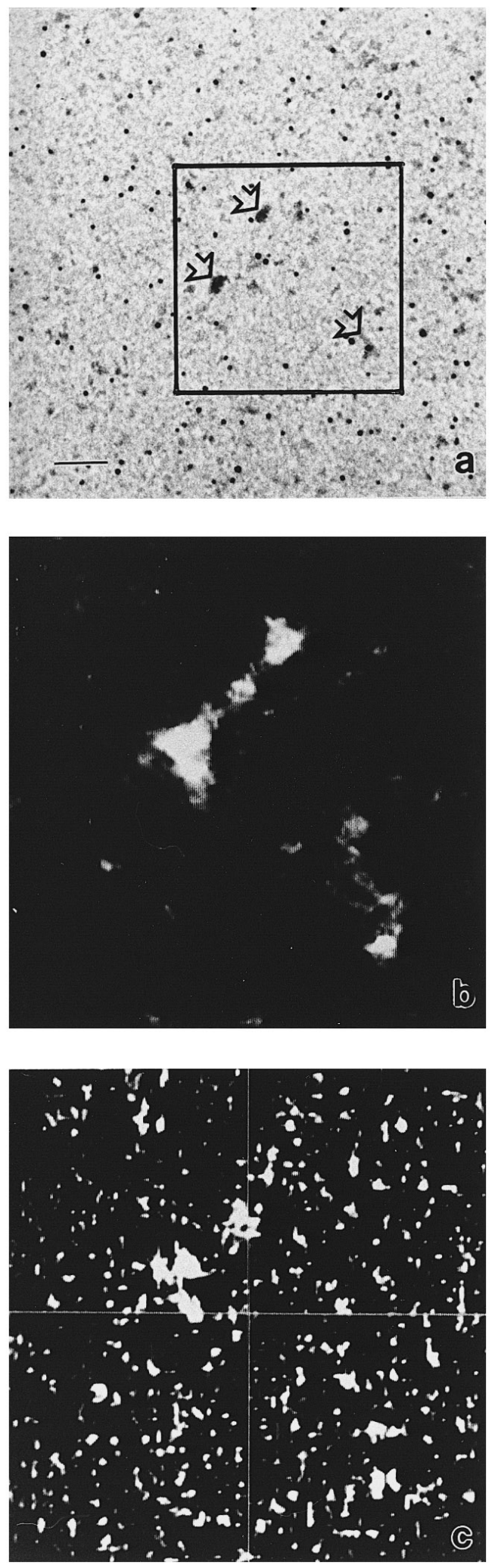

FIG. 3. Conventional imaging and reconstructed images of cytoplasm with three large precipitates (open arrows) and small precipitates associated with proteins. (a) Conventional imaging of the intercellular precipitates (open arrows). The frame indicates which part of the section is reconstructed; scale: $100 \mathrm{~nm}$; (b) Reconstructed simulated electron microscopic image of the whole reconstructed volume. (c) XY plane through the reconstructed image demonstrating the large precipitate running through the whole thickness of the branes and (c) considering the relatively small amount of these calcium binding proteins in the lens it is difficult to imagine their accounting for the bulk binding of calcium in the lens. Physical-chemical studies (Sharma et al., 1989; Sharma and Balasubramanian, 1996) have given evidence of $\beta$ crystallins binding calcium albeit with a low $\mathrm{K}_{\mathrm{d}}$. Their distribution through the cytoplasm and their abundancy make them possible candidates but more physical-chemical data about their binding properties, especially in highly concentrated protein environments are needed as definitive proof.

The fact that these tiny precipitates are indeed formed and are not subject to recrystallization and redistribution, suggested often in reviews as a serious drawback of the OPA technique (Mentré and Escaig, 1988; VonZglinicki and Punkt, 1986; Suzuki and Sugu, 1989; VanIren et al., 1979), may be due to the exceptionally high protein concentration (30-40\%) in the lens fibres. Although such matrix conditions do not favour the onset of a precipitation reaction, they are known to inhibit diffusion of the final reaction product before its precipitation and to reduce resolubilization and redistribution of once formed precipitate (VanDuijn, 1991) and consequently, they improve the localization.

Occasionally large pyroantimonate precipitates are encountered in the fibre cytoplasm. They are not associated with any kind of organelle, nor with conspicuous amounts of protein. In the reconstructed sections it was observed that in many cases the amount of small precipitates in the immediate vicinity of these large precipitates is diminished. Therefore we tentatively conclude that these large precipitates result from a local reaggregation of the small precipitates, perhaps as a consequence of irregularities in the protein matrix of the fibre cytoplasm. This assumption is supported by the observation that DMSO treated lenses reveal a considerably increased frequency of these large precipitates (Vrensen and Sanderson, unpublished observations). DMSO affects the hydration state of proteins and makes $\mathrm{Ca}^{2+}$ ions available for precipitation in large aggregates.

Our findings are a further indication that the maintenance of membrane integrity and function is of great importance in the lens. It separates the interand intra-cellular space, since both disturbance of the equilibrium between the different calcium stores and the presence of intracellular free calcium will in-

section as well as the small highly scattering precipitates often associated with the less scattering proteins.

Note: Owing to computer presentation of the results, mass in the reconstructed cross sections is invariably represented as white, the absence of mass as black. $\mathrm{X}$ and $\mathrm{Y}$ indicate the orientation of the object plane in the electron microscope, $\mathrm{Z}$ the direction of the electron optical axis (i.e. perpendicular to the XY plane). 
evitably lead to cataractous changes and finally to cataract. The magnitude of the calcium gradient over the lens fibre membrane is difficult to assess since no data are available on the intercellular calcium concentration. To the best of our knowledge no straightforward techniques exist for a direct measurement of the calcium concentration in the intercellular cleft and the data provided by Duncan and Jacob (1984) relate to the cytoplasmic concentration of ionic calcium as measured with calcium sensitive electrodes.

\section{Acknowledgements}

The authors wish to thank P. Swart for constructing an interface for the goniometer stage of the EM420 Philips electron microscope which considerably facilitated recording of the tilt series and they thank Dr E. Pels and her coworkers from the Corneabank for the human lenses; the human eyes being donated for transplantation purposes. We thank the donor and his family for their generosity and we thank the BIS-foundation (Leiden, NL) for its assistance in obtaining the tissue.

This study was carried out under the auspices of the European Union Concerted Action on the Role of Cell Membranes in Lens Ageing and Cataract (BMH1-CT931650).

\section{References}

Basnett, S. and Beebe, D. C. (1992). Coincident loss of mitochondria and nuclei during lens fiber differentiation. Dev. Dynamics 194, 85-93.

DeWolf, A. and Vrensen, G. F. J. M. (1995). Calcium distribution in the human lens. Vis. Res. 35 (S), 196.

Duindam, J. J., Vrensen, G. F. J. M., Otto, C., Puppels, G. J. and Greve, J. (1995). New approach to assess the cholesterol distribution in the eye lens: confocal Raman microspectroscopy and filipin cytochemistry. J. Lipid Res. 36, 1139-46.

Duindam, J. J., Vrensen, G. F. J. M., Otto, C. and Greve, J. (1996). Aging affects the conformation of cholesterol in the human eye lens. Ophthalmic Res. 28 (S1), 86-96.

Duncan, G. and Jacob, T. J. C. (1984). Influence of external calcium and glucose on internal total and ionized calcium in the rat lens. J. Physiol. 357, 485-93.

Duncan, G., Williams, M. R. and Riach, R. A. (1994). Calcium, cell signalling and cataract. Prog. Ret. Eye Res. 13, 623-52.

Frank, J. (1992). (Ed.) Electron tomography: threedimensional imaging with the transmission electron microscope. Pp. 1-39. Plenum Press: New York, U.S.A.

Jonges, R., DeMoor, E., Boon, P. M. N., VanMarle, J., Dietrich, A. J. J. and Grimbergen, C. A. (1996). Three-point repositioning of axis; a three dimensional alignment procedure for electron microscope tomography using three markers. Micro. Res. Tech. 33, 516-26.

Li, L. K., So, L. and Spector, A. (1985). Membrane cholesterol and phospholipid in consecutive sections of human lenses. J. Lipid Res. 26, 601-9.

Mentré, P. and Escaig, F. (1988). Localization of cations by pyroantimonate. I. Influence of fixation on distribution of calcium and sodium. J. Histochem. Cytochem. 36, 49-54.

Prescott, A., Duncan, G., VanMarle, J. and Vrensen, G. (1994). A correlated study of metabolic cell communication and gap junction distribution in the adult frog lens. Exp. Eye Res. 58, 737-46.

Sharma, Y. and Balasubramanian, D. (1996). Calcium binding properties of $\beta$-crystallins. Ophthalmic Res. 28 (S1), 44-7.

Sharma, Y., Rao, C. M., Narasu, M. L., Somasundaran, T., Gopalakrishna, A. and Balasubramanian, D. (1989). Calcium ion binding to delta- and beta-crystallins: The presence of the 'EF-hand' motif in delta-crystallin that aids in calcium ion binding. J. Biol. Chem. 264, 12794-9.

Suzuki, S. and Sugi, H. (1989). Evaluation of pyroantimonate methods for detecting intracellular calcium localization in smooth muscle fibres by X-ray microanalysis of cryosections. Histochemistry 92, 95-101.

VanDuijn, P. (1991). Model systems. Principles and practice of the use of matrix-immobilized enzymes for the study of the fundamental aspects of cytochemical enzyme methods. In Histochemistry, Theoretical and Applied. (Eds Stoward, P. J., Everson Pearse, A. G.) Churchill Livingstone. Vol. 3, Pp. 433-72.

VanIren, F., VanEssen-Jolen, L., VanderDuyn-Schouten, P., Boers-VanderSluijs, P. and DeBruijn, W. C. (1979). Sodium and calcium localization in cells and tissues by precipitation with antimonate: a quantitative study. Histochemistry 63, 273-94.

VanMarle, J., Vrensen, G. and VanVeen, H. (1991). Maturing human eye lens fibre membranes and filipin cytochemistry. In Eye lens membranes and aging. (Eds Vrensen, G. F. J. M. and Clauweaert, J.) Proceedings of the EURAGE Symposium. Pp. 123-34.

VanMarle, J., Dietrich, A. J. J., Jonges, K., Jonges, R., DeMoor, E., Vink, A., Boon, P. and VanVeen, H. (1995). EM tomography of section collapse, a non-linear phenomenon. Micro. Res. Tech. 31, 311-16.

VanReempts, J., Borgers, M. and Offner, F. (1982). Ultrastructural localization of calcium in the rat retina with a combined oxalate-pyroantimonate. Histochem. J. 14, 517-22.

VonZglinicki, T. and Punkt, K. (1986). Localization artefacts in ultracytochemical ion precipitation reactions. Histochem. J. 18, 29-35.

Vrensen, G. F. J. M. and DeWolf, A. (1996). Calcium distribution in the human eye lens. Ophthalmic Res. 28 (S2), 78-85.

Vrensen, G. F. J. M., VanMarle, J., VanVeen, H. and Willekens, B. (1992). Membrane architecture as a function of lens fibre maturation: A freeze fracture and scanning electronmicroscopic study in the human lens. Exp. Eye Res. 54, 433-46.

Vrensen, G. F. J. M., Sanderson, J., Willekens, B. and Duncan, G. (1995). Calcium localization and ultrastructure of clear and pCMPS treated rat lenses. Invest. Ophthalmol. Vis. Sci. 36, 2287-95. 